\title{
Agent-based Dynamic Model for Pedestrian Counter Flow
}

\author{
PEIHONG ZHANG ${ }^{1}$, XIAOYAN HUANG $^{1}$, SIUMING LO ${ }^{2}$, MAOHUA ZHONG $^{3}$, \\ HUANHUAN WAN ${ }^{1}$, and MU LIU ${ }^{1}$ \\ ${ }^{1}$ Faculty of Environment Engineering \\ Shenyang Jianzhu University \\ Hunnan new district of Shenyang \\ Liaoning, P.R. China, 110168 \\ ${ }^{2}$ Department of Building and Construction \\ City University of Hong Kong \\ ${ }^{3}$ China Academy of Safety Science and Technology \\ 17 Huixin Xijie \\ Beijing, P.R. China, 100029
}

\begin{abstract}
This paper presents the establishment of the Agent-based Dynamic Model for Pedestrian counter flow (ADMP). It was shown that a rule set of pedestrian behaviors and its combination with a dynamic model for pedestrian flow was capable of effectively capturing the crowd flow rules of pedestrians using agent-based technology. Two digital tests of bottlenecks of evacuation passage in counter flow are presented, in which 200 and 300 pedestrians were stochastically distributed in a space measuring $40000 \times 10000$ $(\mathrm{mm})$. Crowd behavior phenomena, such as arching, jamming, lane forming, and oscillating could be observed after certain critical points. From a comparison of the two digital tests, it could be seen that, with an increase in pedestrian density, the counter flow became more and more disorderly, and the two different directions of flow struggled more excitedly as a result. It illustrated that the ADMP was efficient in the simulation of pedestrian flow and is a useful tool for fire safety research and management.
\end{abstract}

KEYWORDS: agent technology, human behavior, counter flow, arching, lane, oscillation

\section{INTRODUCTION}

From the early 1990's until recently, high-rise buildings and densely populated occupancies have become the major feature of new constructions in China. On the other hand, normal fire protection codes are not strictly and completely obeyed in the refurbishment and reconstruction of existing buildings for commercial reasons. The fire hazard associated with these buildings is becoming more and more serious. On account of the large occupancy in buildings, crowd disasters, in terms of multiple death and injury, have become one of the main risks in building fire. In emergency situations, such as fire, occupants always tend to follow preceding pedestrian crowds and select the relatively familiar route to evacuate. As a result, a huge number of evacuees congest at certain exits and crowd disasters could result.The 9/11 incident in the USA was not the only disaster that attracted the attention of government to human evacuation in fire. The fires in the Garley Building (40 deaths), Top-One Karaoke (15 deaths), Luoyang Dongdu Commercial Building (309 deaths) all attracted great attention with respect to human evacuation from almost every social group, including government, fire fighters, planners, engineers and building occupants. 
Many scholars, including Fruin [1], Predtechenskii and Milinskii [2], have conducted extensive observations and analysis of pedestrian flow. Their research has been referred to in the traditional fire prevention codes around the world and also in the newly developing performance-based fire safety codes. Apart from scenario observations, Thompson and Marchant [3] conducted experiments to simulate pedestrian flow and their interactions. Recently, researchers have developed several computational simulation models to determine pedestrian flow rules. Hughes analogized pedestrian flow as a continual flow of fluid or compressible gas using Navier-Stokes equations [4]. However, pedestrian flow is quite different from fluid in that pedestrians are discrete in low densities and have subjective initiative. The fluid-analogized model could only be applied in high density conditions (more than 4 person per square meter) where evacuees could not make their own decisions and could only act as a group and in continuity. Following the successful application of a Cellular Automata (CA) [5-14] model in the vehicle transportation research field, researchers extended its application to the field of pedestrian flow. Based on evacuation space grids and some probabilistic rules of human behavior with respect to exit route choice, CA models have fast run times and could be designed to trace escaping personnel. However, pedestrian flow is more complex and quite different from vehicles in several aspects. Firstly, pedestrian walkways are not as strictly regulated as vehicle roadways; pedestrians are free, stochastic and able to change their walking speed and direction when available gaps could satisfy their subjective necessity. Meanwhile, pedestrian are compressible. Slight bumping and nudging are often characteristics of walking through crowded corridors. Injury is not really an issue if the congestion density and the last congestion time do not exceed certain critical limits. Pedestrian flow is more dangerous than vehicles in that, in emergency situations, pedestrians may rush to egress without any limitations such as vehicle size or channel. Another problem is that the CA model prescribes the pedestrian flow along a pre-assigned network, and the simulation results largely depend on the human behavior rules, which are extremely difficult to define. A social force model by Helbing $[15,16]$ simulated pedestrian flow based on self-driven forces and panic psychology. However, it is quite difficult to quantify the inter-pressure and the frictional force between pedestrians, and between pedestrians and building structures, such as walls, barriers, etc.

Bottlenecks in walkways due to bi-directional counter flow present one of the most dangerous situations that could result in crowd disaster. Based on crowd dynamic analysis of pedestrian flow, we established the Agent-based Dynamic Model for Pedestrian Counter flow (ADMP), where evacuees are modeled as intelligent agents under the direction of behavioral rules. The counter flow pattern going along a corridor was simulated. The pedestrian flow pattern near the bottlenecks was analyzed and discussed further.

\section{AGENT-BASED CROWD DYNAMIC MODEL}

\section{Establishment of the Agent}

Pedestrians are strongly subjective and demonstrate initiative especially in emergency situations. They will actively move towards safety within the available safe evacuation time (ASET) under subconscious least effort and least obstruction rules, etc. In this paper, pedestrians are modeled as agents, who are self-driven objects, for example safety destination driven, least restrained driven, etc. Once they are located in certain surroundings, agents interact with other objects (i.e., other evacuees, wall, obstructions, etc.) under the direction of their own internal physical and personnel behavior characteristics. The internal behavior characteristics can be described by the following 
three aspects: external perception, internal perception and their movement ability [17-20].

External perception: Each agent has the ability to view outside objects and perceive stimulation. In this paper, the effective view angle is assumed to be $200^{\circ}$. The visible distance is not defined here, assuming that agents could see the whole space if no objects obstructs it beforehand. It should be mentioned that the surrounding physical environment parameters, i.e., smoke, have some influence on visible distance and this aspect should be developed in the future, based on experiments or physical tests.

Internal perception: Each agent has his internal perception ability, depending on his behavioral characteristics, i.e., patience, strong desire for safety and the least effort rules, etc. When actual speed cannot reach the desired speed, the patience psychology will be challenged with the congesting status lasting. Pedestrians become bellicose and crowded with each other, the flow ability of egress decreases, and the pedestrians will congest near bottlenecks i.e., exits, stairs, etc. Once the congestion time increases beyond the critical time, the possibility of crowd death and injury will increase. The desire for safety contributes to the agent's desired speed of movement and the degree of patience in the congestion areas. The least effort rules will contribute significantly to his route-selecting process. In this paper, the internal perception ability was simulated based upon if-then rules and logic analysis.

\section{Dynamic Model of the Agent}

Agents were modeled as soft and elastic circle objects, with interactive force and clear boundary, moving to a distinctive destination towards safety egress or a refuge space. The dynamic model of agents was established as follows [21]:

$\vec{F}_{i}=\vec{F}_{i, s}+\vec{G} \cos \alpha+\sum_{i \neq j} \vec{F}_{i, j}+\sum_{\text {wall }} \vec{F}_{i, w}+\vec{F}_{i, o b s t r u c t}$

where $\vec{F}_{i}$ refers to the joint force vector exerted on the agent $i$; $\vec{F}_{i, s}$ refers to the self-driven force of pedestrians; $\vec{G} \cos \alpha$ is the divided force of gravity $\vec{G}$, where the angle between $\vec{G}$ and the walkway is $\alpha$; $\vec{F}_{i, j}$ refers to the inter-active force between different agents $i$ and $j$; the force between agent $i$ and the wall is defined as $\vec{F}_{i, w}$, and the force from obstructions $\vec{F}_{i, o b s t r u c t}$. The agent would adjust his walking velocity and make his way-selecting decision under the effect of the joint force $\vec{F}_{i}$. The gravity forces exerted on agents can be positive in stairs descent as illustrated in Fig. 1.

The self-driven force $\vec{F}_{i, s}$ refers to the force vector directed to the safety refuge destination region. When approaching the egress destination, evacuees will most probably strive simultaneously for the final safety place without any negotiation. Once the congestion status increases above a certain critical density $\rho_{h}$ and critical time $t_{h}$, pedestrians will lose their patience and crowd disasters can escalate. With time passing, congestion density decreases gradually. When the congestion density decreases to the lower limit of $\rho_{l}$, pedestrians will spontaneously adjust their evacuation action with the purpose of flowing more fluently, i.e., forming a lane, etc. The spontaneous adjustment of the evacuation behavior was realized using logic behavior rules (if-then) for agents. 


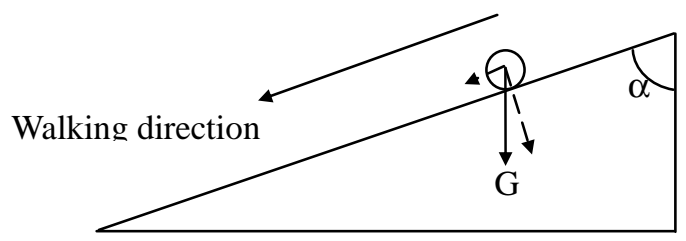

Fig. 1. Gravity and its effect on pedestrian flow down stairs.

The self-driven force $\vec{F}_{i, s}$ largely depends upon the behavioral character of pedestrians, the hazardous status of their surroundings, as well as the local pedestrian density. It is assumed to be a function of desired speed $\vec{V}_{i}^{0}(t)$, which refers to the desired speed of any agent $i$ at any time $\tau_{i}$ in the process of evacuation [21]:

$\vec{F}_{i, s}=m_{i} \frac{\vec{V}_{i}^{0}(t)-\vec{V}_{i}(t)}{\tau_{i}}$

where $\vec{V}_{i}^{0}(0)$ refers to the initial desired speed at start time $\tau=0, \vec{V}_{i}^{0}(t)$ refers to the desired speed at any time $\tau_{i}, V_{i}(t)$ refers to the actual velocity in the desired movement direction. The evacuation direction depends upon the human behavior and the joint efforts $\vec{F}_{i}$.

The interactive force between agents $\vec{F}_{i, j}$, and the force $\vec{F}_{i, w}$ can be divided into tangential force and normal force. Tangential force refers to the friction between agents and with obstructions such as a wall, depending on the facial characteristic parameter of agents and objects in the surroundings. Normal force refers to the pressure between agents and objects, depending on the relative distance between them, which allows compression with each other. $\vec{F}_{i, j}$ and $\vec{F}_{i, w}$ can be expressed as follows:

$\vec{F}_{i, j}=-k \delta_{i j} \vec{r}_{i j}$

$\vec{F}_{i w}=-k \delta_{i w} \vec{r}_{i w}$

where, $\vec{r}_{i j}$ and $\vec{r}_{i w}$ refers to the displacement vector from the center of agent $i$ to agent $j$ or the wall, $\delta_{i j}$ and $\delta_{i w}$ are the size of the interactive force between agents $i, j$ and the wall. Many researchers have conducted much research on this. Helbing[15,16] believed that it had a power relationship with the distance between $i, j$ and the wall, and this was what we adopted here. $k$ is the elastic constant and a function of the degree of their approaching or receding, reflecting that agents are compressible. Equation 5 refers to the relationship of the elastic constant between the two states, i.e., receding or approaching, where the value of $\beta$ is less than 1 and depends on the patience behavior characteristic of agents. Equation 5 is equivalent to a compensatory coefficient, which could prevent the agents from bumping into each other like rubber balls. 


\section{SIMULATION AND DISCUSSION}

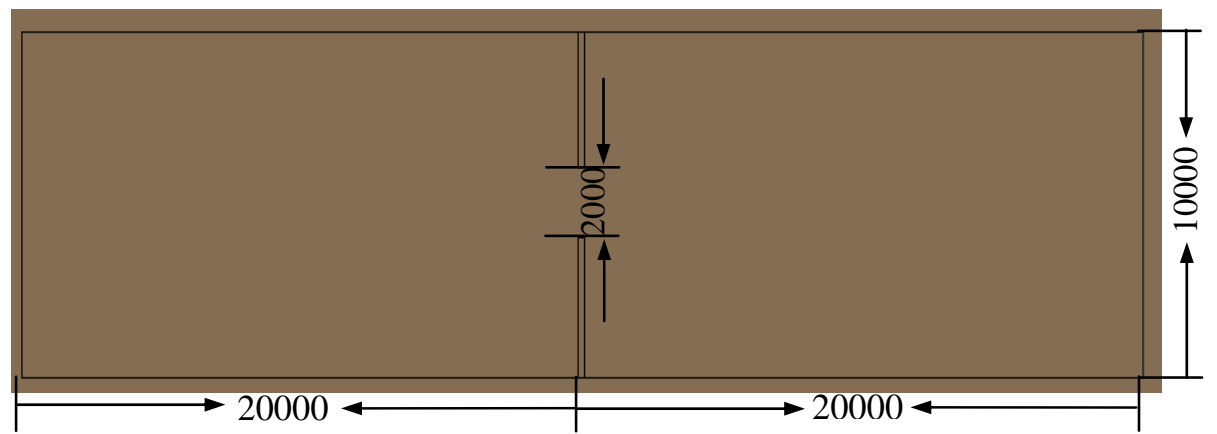

Fig. 2. Spatial dimension of the digital testing walkway.

Using the agent-based dynamic model, pedestrian counter flow was simulated in the digital test space illustrated in Fig. 2. At the initial simulation time, a total of 200 persons were stochastically distributed in the $40000 \mathrm{~mm} \times 10000 \mathrm{~mm}$ walkway. There was a $2000 \mathrm{~mm}$ width door in the middle part of the walkway. Pedestrians walked in a counter flow urgently at an initial desired velocity of $2.0 \mathrm{~m} / \mathrm{s}$. Pedestrians moving towards the left were represented as a green circle; on the opposite walkway, pedestrians moving towards the right were illustrated as a yellow circle. The mean shoulder width of pedestrians was presumed as $0.45 \mathrm{~m}$. The simulation scenarios of the 200 pedestrians are shown in Figs. 3-8.

From the simulation, it could be seen that, under the strong desire for safety refuge or some other stimulation, pedestrians strived anxiously towards their destination without negotiation with each other. The following crowd dynamic characteristics were highlighted:

1) Arching: After a certain critical time and critical density, arching could be observed near the door. Pedestrians crowded near the door with arching boundary layers, whereby each pedestrian occupied the optimum location and was the same distance from the center point of the egress. This was consistent with the behavioral characteristic of pedestrians, where they were modeled as agents with artificial intelligence under the direction of least effort and shortest walking distance (see Fig. 4).

2) Jamming: At increased densities, jamming phenomena could occur when many people tried to flow through the bottleneck of the walkway (i.e., door) at the same time. This clogging was especially typical of a bi-directional flow situation where two groups of pedestrians blocked each other without any negotiation.

3) Lane forming: The jamming status lasted for a certain period of time until a lane could form spontaneously by self-organization under certain leadership characteristics of pedestrians (i.e., the leader was sufficiently strong). In this way, strong interactions with oncoming pedestrians were reduced and a higher walking speed became possible. However, the lane could be broken easily and frequently by the opposite flow of pedestrians in emergency situations where there was no order 
and plan for the whole evacuation process (see Fig. 8).

4) Oscillations: At bottlenecks in the counter flow, e.g., doors, oscillation phenomena could be observed in the digital test. Once a pedestrian was able to pass the bottleneck, it became easier for others to follow him in the same direction until the point when somebody was able to pass the bottleneck in the opposite direction. The initially left-directional pedestrian flow could emerge out of the bottleneck (see Figs. 4-6) first. Approximately 24 seconds later, the right-directional pedestrian flow emerged and formed a lane directly towards the right. Sometimes bi-directional pedestrian flow came face to face just near the door and two lanes were formed simultaneously as shown in Fig. 8, after 50.5 seconds of evacuation. This illustrated that the two divisions of the counter flow produced, to a certain extent, a kind of balance. It should be mentioned that the direction of flow which emerged first was determined by the crowd behavior of pedestrians and could in fact show up stochastically in different test cases.

Another simulation was performed in which 300 pedestrians were initially and stochastically distributed in the same walkway with the same initial conditions. From the comparison of the two digital tests, it could be seen that, with the increase in pedestrian density, the counter flow became more and more disorderly, and the two different directions of flow struggled more excitedly as a result (see Fig. 9-10).

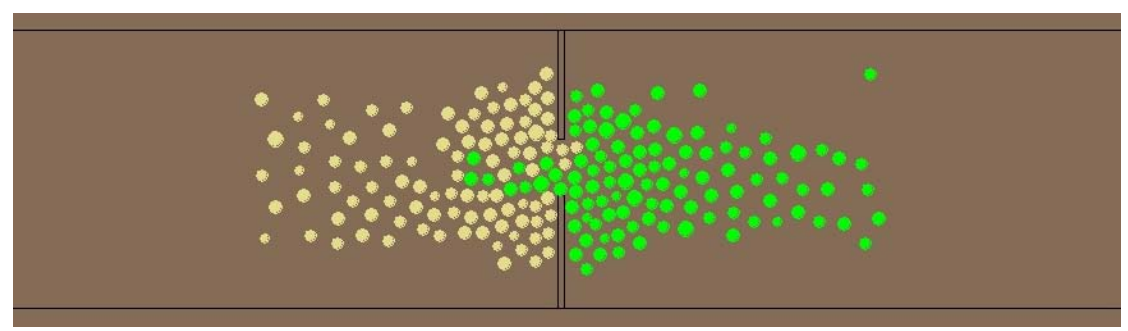

Fig. 3. $\mathrm{t}=4.5 \mathrm{~s}$.

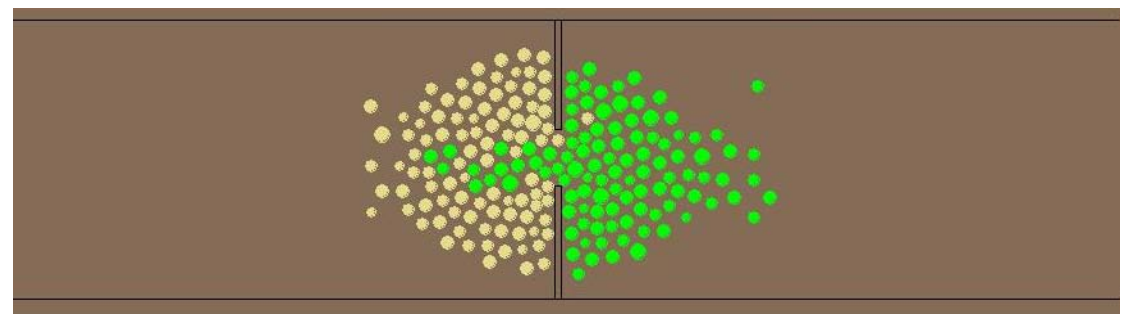

Fig. 4. $\mathrm{t}=6.5 \mathrm{~s}$.

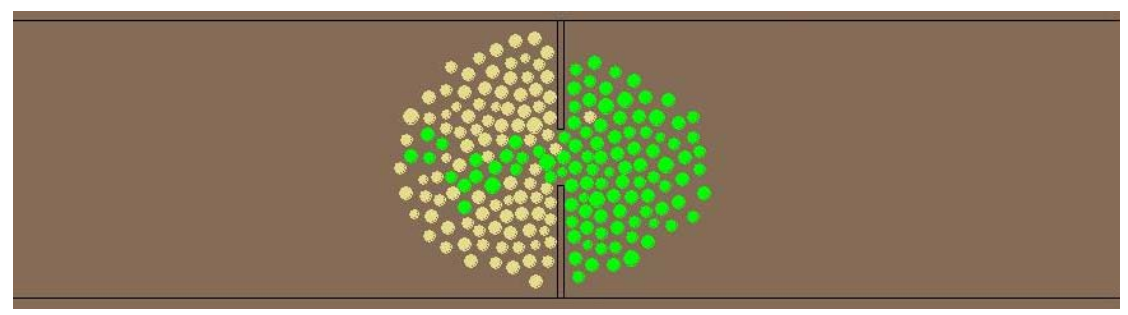

Fig. 5. $\mathrm{t}=8.0 \mathrm{~s}$. 


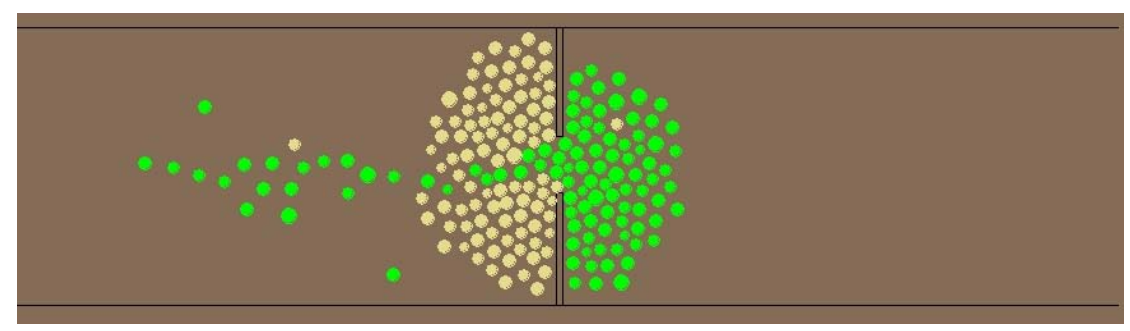

Fig. 6. $\mathrm{t}=12.5 \mathrm{~s}$.

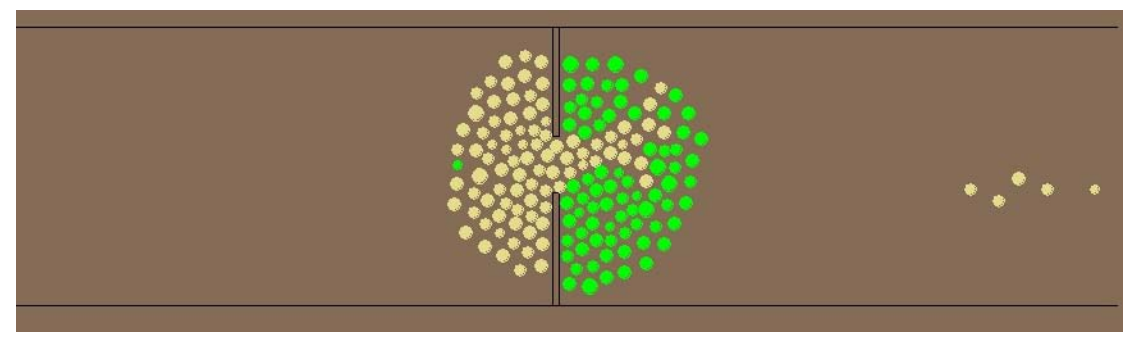

Fig. 7. $\mathrm{t}=36.7 \mathrm{~s}$.

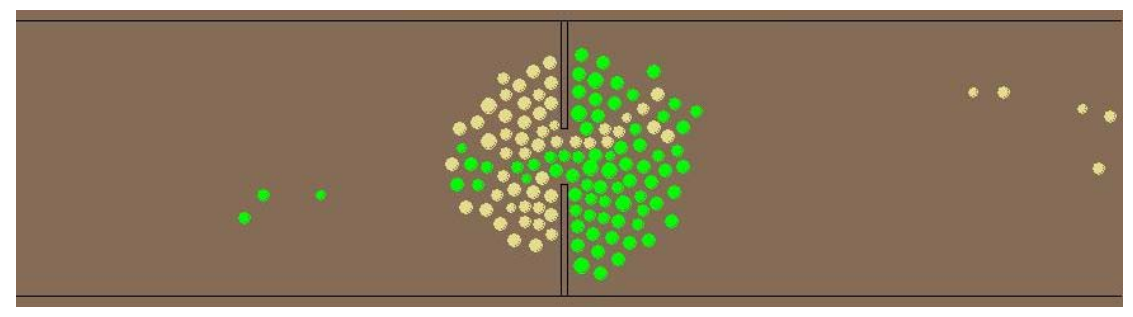

Fig. 8. $\mathrm{t}=50.5 \mathrm{~s}$.

\section{DISCUSSION AND CONCLUSION}

Pedestrian flow is a strongly interacting system of many persons. Based on the lattice-gas model, Itoh and Nagatani [11] et al., did some theoretical research and simulation of the moving of an audience between two halls to avoid the jammed state of pedestrians in rush hour, panic escape, etc. (see Fig. 11). It was found that there existed an optimal admission time for moving the audience, and the optimal moving was related to the jamming transition in the pedestrian counter flow. When the admission time was less than the optimal time, the jammed state appeared near the gate and the visitors could not enter the inner hall because the outflow of walkers prevented the inflow of walkers from entering. The simulation result was to some extent similar to our research finding with respect to jamming discussed earlier.

Based on a behavioral force model, Helbing interpreted the collective patterns of motion as a self-organization phenomena arising from the nonlinear interactions between pedestrians, which are caused by environmental changes and their influence on human walking behavior. Helbing [22] also found oscillation of the passing direction at narrow passages (see Fig. 12.), which could support the validity of our research result to some extent.

From the discussion above, it can be seen that the agent-based dynamic model for pedestrian flow (ADPM) could be applied in the simulation of pedestrian counter flow. 


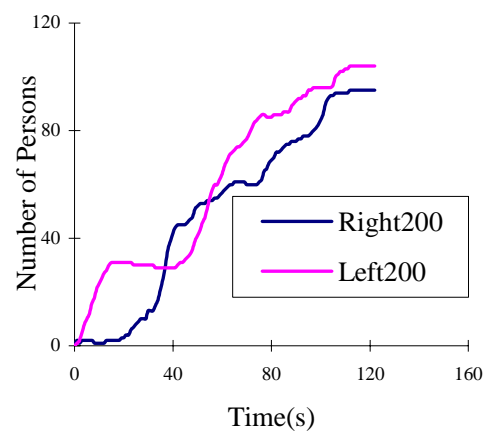

Fig. 9. 200 persons stochastically distributed in two rooms and moving in counter flow, $\mathrm{v}^{0}(0)=2.0 \mathrm{~m} / \mathrm{s}$.

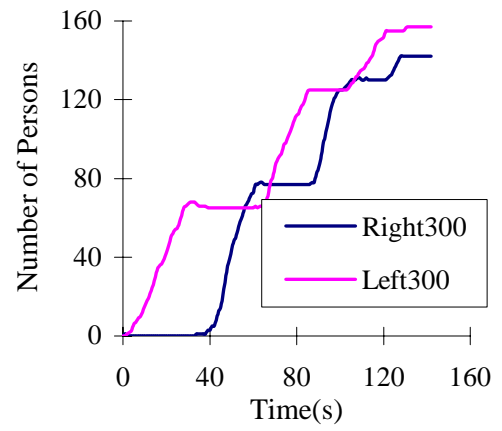

Fig. 10. 300 persons stochastically distributed in two rooms and moving in counter flow, $\mathrm{v}^{0}(0)=2.0 \mathrm{~m} / \mathrm{s}$.
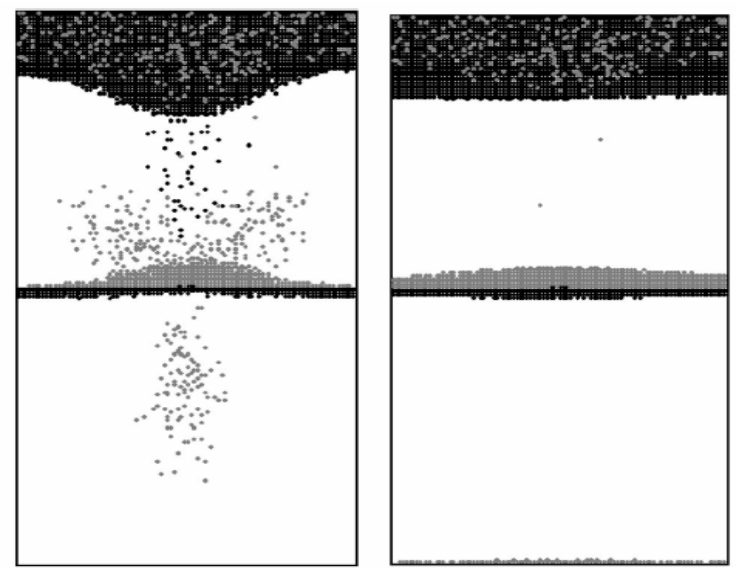

Fig. 11. Itoh and Nagatani's research on the shifting of the audience [11].

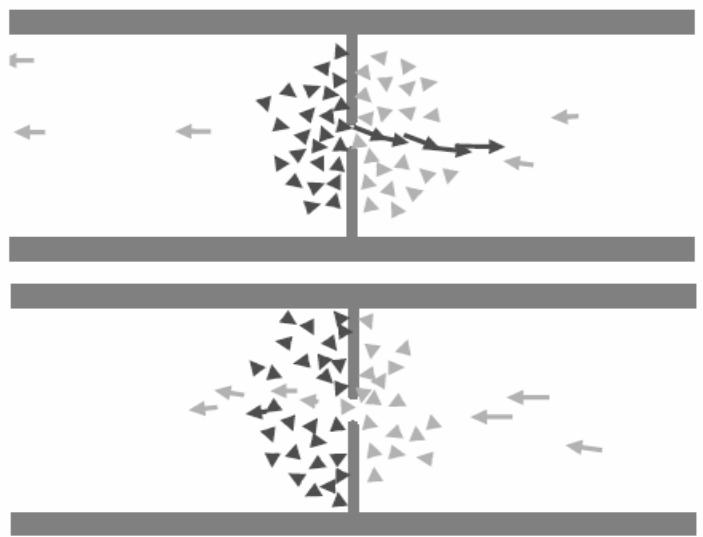

Fig. 12. Helbing's research on the oscillation of the passing direction at narrow passages [22]. 
The main feature of this model is the behavioral rules assigned to the agents of artificial intelligence. On the other hand, the dynamic force model of pedestrian flow needs to be updated further, especially on the relevant parameters relating to the interactive force between agents, agents and the objects, and the self-driven pedestrian force. The number of relevant parameters should be discussed further to satisfy the different status of pedestrians' crowded behavior. Arching and clogging of people occur at the bottleneck of the walkway. Lanes could be formed spontaneously and the oscillation phenomenon could be observed. Further research needs to be performed in real buildings or experimental scenarios to further develop and validate the model.

\section{ACKNOWLEDGEMENT}

This paper is funded by the NSFC research project of P.R.C entitled as "Pedestrian Flow Study in Densely Populated Evacuation Space (70303016)”, and China National Key Basic Research Special Funds (Grant No. 2001CB409600).

\section{REFERENCES}

[1] Fruin, J.J, Service Pedestrian Planning and Design, MAUDEP, 1971, 1987.

[2] Predtechenskii, V.M and Milinskii, A.I, Planning for Foot Traffic Flow in Buildings, Amerind Publishing Co., 1978.

[3] Thompson, P.A. and Marchant, E.W., Modeling Techniques for Evacuation. Engineering for Crowd Safety, Elsevier Science Publishers B.V., 1993, p. 259-269.

[4] Hughes, R.L., "A Continuum Theory for the Flow of Pedestrians," Transportation Research Part B 36: 507-535 (2002).

[5] Blue, V.J. and Adler, J.L., "Cellular Automata Microsimulation for Modeling Bi-Directional Pedestrian Walkways," Transportation Research Part B 35: 293-312 (2001).

[6] Nagatani, T., "Dynamical Transition in Merging Pedestrian Flow Without Bottleneck," Physica A 307: 505-515(2002).

[7] Fuentes, M.A. and Kuperman, M.N., "Cellular Automata and Epidemiological Models with Spatial Dependence,” Physica A 267: 471-486 (1999).

[8] Tajima, Y. and Nagatani, T., "Clogging Transition of Pedestrian Flow in T-Shaped Channel,” Physica A 303: 239-250 (2002).

[9] Lo, S.M., Fang, Z. et al., "Use of a Modified Network Model for Analyzing Evacuation Patterns in High-Rise Buildings," Journal of Architectural Engineering: 21-29 (2001).

[10] Lo S.M. and Fang Z., “A Spatial-grid Evacuation Model for Buildings,” Journal of Fire Science 18: 376-394 (2000).

[11] Itoh, T. and Nagatani, T., "Optimal Admission Time for Shifting the Audience," Physica A 313: 695-708 (2002).

[12] Isobe, M., Adachi, T. and Nagatani, T., "Experiment and Simulation of Pedestrian Counter Flow,” Physica A 336: 638-650 (2004). 
[13] Gwynne, S., Galea, E.R., Owen, M. et al., "A Review of the Methodologies Used in the Computer Simulation of Evacuation from the Built Environment," Building and Environment 34: 741-749(1999).

[14] Gwynne, S., Galea E.R., Owen, M., and Lawrence, P.J, “An Investigation of the Aspects of Occupant Behavior Required for Evacuation Modeling,” Evacuation from Fires, Volume $2^{\text {nd }}$, Baywood Publishing Company, Inc., Amityville, New York, 2000.

[15] Helbing, D., "Active Walker Model for the Formation of Human and Animal Trail Systems," Physical Reviews E 56: 531-545(1997).

[16] Helbing, D., "Micro- and Macro-Simulation of Freeway Traffic," Mathematical and Computer Modeling, 35: 517-547(2002).

[17] Hayes N., Introduction to Cognitive Processes, The British Psychological Society, London, 1991, p. 23-154.

[18] Bryan J.L., "Behavior Response to Fire and Smoke,” The SFPE Handbook of Fire Protection Engineering ( $\left.2^{\text {nd }} E d\right)$, DiNenno P.J. (ed.), National Fire Protection Association, Quincy, MA, 1995, pp. 241-262.

[19] Canter D., "Studies of Human Behaviour in Fire: Empirical Results and Their Implications for Education and Design,” Building Research Establishment Report, Herts: Fire Research Station, 1985, 1-20p.

[20] Sime J., "Human Behaviour in Fires - Summary Report,” Report No. 45, Building Use \& Safety Research Unit, School of Architecture, Portsmonth Polytechnic, UK. 1992. 385-407p.

[21] ZHANG P. et al., "Behavior Rules of Human Evacuation in Fire," Journal of Northeastern University, 22: 54-57 (2001).

[22] Helbing, D. et al., "Self-organizing Pedestrian Movement," Environment and Planning B 28: 361-383 (2001). 